Marquette University

e-Publications@Marquette

Biomedical Engineering Faculty Research and

Publications

Engineering, College of

$10-1-2013$

Effect of Tilt Sensor versus Heel Loading on Neuroprosthesis Stimulation Reliability and Timing for Individuals Post-Stroke during Level and Non- Level Treadmill Walking

M. Barbara Silver-Thorn

Marquette University, barbara.silver-thorn@marquette.edu

Published Version. International Journal of Physical Medicine and Rehabilitation, Vol. 1, No. 4 (October 2013). DOI. (C) OMICS Publishing Group, 2013.This is an open-access article distributed under the terms of the Creative Commons Attribution License, which permits unrestricted use, distribution, and reproduction in any medium, provided the original author and source are credited. 
This is an open-access article distributed under the terms of the Creative Commons Attribution License, which permits unrestricted use, distribution, and reproduction in any medium, provided the original author(s) and source are credited.

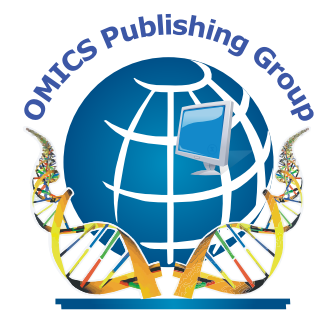

ISSN: 2329-9096

\section{International Journal of Physical Meclicine \& Rehabilitation}

The International Open Access

International Journal of Physical Medicine \& Rehabilitation

\section{Editorial Board}

John Stone

University at Buffalo, USA

Joe Edward Springer

University of Kentucky, USA

Masayoshi Yamaguchi

Baylor College of Medicine, USA

Carmen M. Terzic

Mayo Clinic, Minnesota, USA

Jin J Luo

Temple University School of Medicine, USA

Available online at: OMICS Publishing Group (www.omicsonline.org)

7 his article was originally published in a journal by OMICS

Publishing Group, and the attached copy is provided by OMICS Publishing Group for the author's benefit and for the benefit of the author's institution, for commercial/research/educational use including without limitation use in instruction at your institution, sending it to specific colleagues that you know, and providing a copy to your institution's administrator.

All other uses, reproduction and distribution, including without limitation commercial reprints, selling or licensing copies or access, or posting on open internet sites, your personal or institution's website or repository, are requested to cite properly. 


\title{
Effect of Tilt Sensor versus Heel Loading on Neuroprosthesis Stimulation Reliability and Timing for Individuals Post-Stroke during Level and Non- Level Treadmill Walking
}

\author{
M. Barbara Silver-Thorn*, Michelle B Gallagher and Jason T Long
}

Marquette University, Biomedical Engineering, 1637 West Wisconsin Avenue, Room 313, Milwaukee, WI 53233, USA

\begin{abstract}
Study background: Non-level walking may adversely affect stimulation of neuroprostheses as initial programming is performed during level walking. The objectives of this study were to assess stimulation reliability of tilt and heel sensor-based neuroprosthesis stimulation during level and non-level walking, examine stimulation initiation and termination timing during level and non-level walking, and determine whether heel or tilt sensor-based stimulation control is more robust for non-level ambulation.
\end{abstract}

Methods: Eight post-stroke individuals with drop foot who were able to actively ambulate within the community were selected for participation. Each subject acclimated to the neuroprosthesis and walked on a treadmill randomly positioned in inclined, level and declined orientations. The primary measures of interest were stimulation reliability and timing.

Results: Statistically significant differences in tilt, but not heel, sensor-based stimulation reliability were observed between level and non-level walking trials. Tilt sensor-based stimulation initiation occurred significantly closer to swing as the treadmill processed from declined to inclined orientations. No statistically significant differences in stimulation reliability or timing were observed between theoretical heel versus clinical tilt sensor-based stimulation control.

Discussion and conclusions: Tilt sensor-based stimulation reliability may be adversely affected by non-level walking. Differences in stimulation initiation timing with tilt sensor-based control during non-level walking may be advantageous as stimulation initiation closer to swing during inclined ambulation may allow for greater ankle plantar flexion to assist with forward progression. Despite a lack of significant differences in stimulation reliability or timing between sensors, theoretical heel sensor-based stimulation control exhibited more consistent stimulation timing with less variability than for tilt sensor-based stimulation during non-level ambulation.

Keywords: Stroke; Orthotic device; Electrical stimulation; Neuroprosthesis; Gait

\section{Abbrevations: AFO: Ankle Foot Orthosis; EMG: Electromyogram \\ Introduction}

Twenty percent of the $6,400,000$ stroke survivors alive today [1] are unable to dorsiflex the ankle [2], causing the foot to drag along the floor during swing. This can limit mobility, increasing instability and risk of tripping or falling [3]. The traditional treatment for foot drop is an ankle foot orthosis that holds the ankle in a neutral position during swing, preventing toe drag. An alternative treatment is a neuroprosthesis that electrically stimulates the common peroneal nerve, activating the ankle dorsiflexors during swing.

Use of a neuroprosthesis was first proposed to treat post-stroke individuals with foot drop in 1961, and today there are three FDA approved neuroprostheses. Two of these neuroprostheses incorporate a heel sensor or foot switch placed in the shoe to define the stimulation periods [4].

The third design uses an accelerometer to measure the angle of the tibia to define the stimulation timing [5]; this design also incorporates an optional heel sensor that may be used as an alternative control signal.

One limitation of prior neuroprosthesis studies [6-12] is that only level walking trials were conducted, although non-level walking surfaces are routinely encountered during household and community ambulation. Gait kinematics for normal individuals vary during ambulation on inclined and declined surfaces [13]. Significant increases in hip flexion, knee flexion and ankle dorsiflexion at heel strike, as well as ankle plantar flexion during toe off, have been observed for inclined walking. Peak knee flexion during swing has also been observed to increase during declined walking [13]. Non-level walking may therefore affect the angle of the tibia and/or heel loading at heel strike and toe off, adversely affecting stimulation for tilt and/or heel sensor-based control of neuroprostheses as initial fitting and programming are performed during level walking.

The objectives of this study are to assess stimulation reliability of tilt and heel sensor-based neuroprosthesis stimulation during level and non-level walking, examine stimulation initiation and termination timing during level and non-level walking, and determine whether heel or tilt sensor-based stimulation control is more robust for non-level ambulation.

\section{Materials and Methods}

Eight post-stroke individuals were selected for participation in this study as per initial a priori power analyses $(\mathrm{N} \geq 7)$. These subjects were at least 6 months post-stroke that resulted in hemiplegia, were able

*Corresponding author: M. Barbara Silver-Thorn, Marquette University, Biomedical Engineering, 1637 West Wisconsin Avenue, Room 313 Milwaukee, WI 53233, USA, Tel: (414)-288-3608; Fax: (414)-288-7938 E-mail: barbara.silver-thorn@marquette.edu

Received August 23, 2013; Accepted October 27, 2013; Published November 02, 2013

Citation: Silver-Thorn MB, Gallagher MB, Long JT (2013) Effect of Tilt Sensor versus Heel Loading on Neuroprosthesis Stimulation Reliability and Timing for Individuals Post-Stroke during Level and Non-Level Treadmill Walking. Int J Phys Med Rehabil 1: 163. doi:10.4172/2329-9096.1000163

Copyright: () 2013 Silver-Thorn MB, et al. This is an open-access article distributed under the terms of the Creative Commons Attribution License, which permits unrestricted use, distribution, and reproduction in any medium, provided the original author and source are credited. 
Citation: Silver-Thorn MB, Gallagher MB, Long JT (2013) Effect of Tilt Sensor versus Heel Loading on Neuroprosthesis Stimulation Reliability and Timing for Individuals Post-Stroke during Level and Non-Level Treadmill Walking. Int J Phys Med Rehabil 1: 163. doi:10.4172/23299096.1000163

Page 2 of 7

to achieve a neutral ankle position passively, had no prior experience with a neuroprosthesis, and were capable of walking 30 meters without the use of a cane or walker and without stopping to rest. Subjects were excluded who experienced a fall within the previous 3 months, a history of seizures, or a Botox injection in the lower limb within the past 6 months. Additional exclusionary criteria included cognitive disability due to stroke, musculoskeletal injuries of the paretic or nonparetic lower limb, pregnancy, or a pacemaker/defibrillator/metallic implant. This study was approved by the Institutional Review Boards of Marquette University and the Medical College of Wisconsin. Written informed consent was obtained prior to subject participation in any research activities.

A physical therapist with extensive neuroprosthesis experience fit and programmed the neuroprosthesis (WalkAide, Innovative Neurotronics, Austin, TX), using tilt-sensor stimulation control, for each subject. The therapist manually stimulated the peroneal nerve as the subject walked within parallel bars, defining the tibial angle thresholds for stimulation initiation and termination. Each subject was instructed to slowly acclimate to the neuroprosthesis, ramping up from 2 to 8 hours of use per day over a one week period and fulltime use within two weeks, as per manufacturer recommendations and clinical practice. The subject then met with the therapist for evaluation and potential adjustments; further adjustment visits were scheduled as needed.

After 4 weeks acclimation, gait analysis was conducted on a treadmill (L8, Landice, Inc., Randolph, NJ), randomly positioned in level, inclined $\left(+7^{\circ}\right)$ and declined $\left(-7^{\circ}\right)$ orientations. For each treadmill orientation, the subject determined his/her comfortable walking speed; two minute walking tasks, inclusive of at least eight 10 second walking trials, were then conducted. No adverse events during acclimation or gait analysis were observed.

The WalkAide heel sensor was placed under the insole of the subject's shoe. An insole (F-scan, VersaTek System, Tekscan, Inc., South Boston, MA), trimmed to the subject's shoe size, was positioned between the insole and foot on the affected side, providing duplicate heel loading data; this insole was calibrated based on the subject's body weight [14]. The neuroprosthesis was positioned after the subject donned their shoes. Reflective markers on the heel and toe were tracked using the Vicon motion analysis system (Vicon 524 Motion Analysis, Vicon Motion Systems Inc., Los Angeles, CA) [14]. The minimum vertical velocity of the heel and toe markers was used to identify heel strike and toe off events, respectively. Bipolar surface electromyographic (EMG) electrodes (MA300, Motion Lab Systems, Inc., Baton Rouge, LA) were positioned over the tibialis anterior of the affected limb to estimate stimulation timing relative to heel strike and toe off events [14].

Tibialis anterior EMG data were acquired via the Vicon A/D system, synchronizing the kinematic $(120 \mathrm{~Hz})$ and EMG $(1800 \mathrm{~Hz})$ data. Plantar pressure data $(50 \mathrm{~Hz})$ were acquired on a separate computer. Tilt and heel sensor data, as well as stimulation data, were acquired (25 $\mathrm{Hz}$ ) using the WalkLink (Innovative Neurotronics, Austin, TX) on a third computer [14].

Theoretical stimulations based on WalkAide heel sensor data were determined to contrast heel versus tilt sensor-based stimulation control [14]. Clinical heel sensor programming requires that the clinician set the heel loading (stimulation termination) and unloading (stimulation initiation) thresholds, adjusting these settings as needed to optimize gait. Theoretical heel sensor-based stimulation approximated these clinical programming procedures. A single threshold, based on a percentage of the range of heel sensor output for the respective subject, was defined to differentiate heel loading and unloading periods [14]. Due to errors with the WalkAide heel sensor for two subjects (S7, S8), theoretical heel sensor-based stimulation was based on F-scan heel loading data for these subjects [14].

To investigate missed and extraneous stimulations for tilt and heel sensor-based stimulation, the stimulation reliability, defined as the number of stimulation periods per gait cycle during the 10 second gait trials, was determined. The mean stimulation reliability and associated variability (e.g. standard deviation) across all 10 second trials for a specific treadmill orientation was calculated for each subject. A stimulation reliability value of one indicates that the WalkAide stimulated just once during each gait cycle; stimulation reliability values less than one reflect missed stimulations and values greater than one indicate that extraneous stimulations occurred.

Tilt sensor-based stimulation initiation and termination timing was evaluated based on anterior tibialis EMG data. Digital signal processing was used to identify stimulation periods and stimulation initiation and termination timing. Specifically, a high pass filter (250 $\mathrm{Hz}, 10$ th order Butterworth) was used to eliminate noise and voluntary muscle contractions. Stimulation initiation and termination timing was based on threshold detection of the linear enveloped [e.g. rectified, low pass filtered (8 Hz, 8th order Butterworth)] EMG [14]. Theoretical heel sensor-based stimulation initiation and termination were based on heel loading data, as described above. The stimulation initiation and termination timing was also evaluated in percent gait cycle [14] Stimulation initiation was expressed relative to the start of swing, with negative values reflecting initiation prior to swing and positive values indicating that stimulation initiation occurred during swing (Figure 1). Stimulation termination was expressed relative to stance; stimulation termination greater than $100 \%$ gait cycle indicates that stimulation extended into early stance of the subsequent gait cycle.

Stimulation reliability and timing were non-normally distributed (Kolmogorov-Smirnov test [15]). Non-parametric Friedman testing was therefore conducted to determine whether stimulation reliability or timing differed significantly ( 0.05 level) with level versus non-level ambulation for both tilt and heel sensor-based stimulations. Posthoc Wilcoxon sign rank tests with Bonferroni correction for multiple comparisons were performed to assess whether such differences occurred between level-inclined or level-declined walking. Wilcoxon rank sum tests were conducted to determine whether stimulation reliability or timing differed significantly ( 0.05 level) between sensors.

\section{Results}

Details regarding the eight post-stroke individuals who completed this study are summarized in Table 1.

The stimulation reliability based on clinical tilt sensor-based stimulation is summarized in Figure 2a for each subject for all treadmill orientations. Differences in tilt-sensor based stimulation reliability between level versus non-level treadmill orientations demonstrated borderline statistical significance $(\mathrm{p}=0.051)$.

The theoretical heel sensor-based stimulation reliability is summarized in Figure $2 \mathrm{~b}$. With the exception of two subjects who exhibited extraneous and/or missed stimulations for non-level walking, theoretical heel sensor-based stimulation reliability was approximately unity for all treadmill orientations and did not differ significantly between level and non-level walking trials.

These stimulation reliability data were contrasted to investigate 
Citation: Silver-Thorn MB, Gallagher MB, Long JT (2013) Effect of Tilt Sensor versus Heel Loading on Neuroprosthesis Stimulation Reliability and Timing for Individuals Post-Stroke during Level and Non-Level Treadmill Walking. Int J Phys Med Rehabil 1: 163. doi:10.4172/23299096.1000163
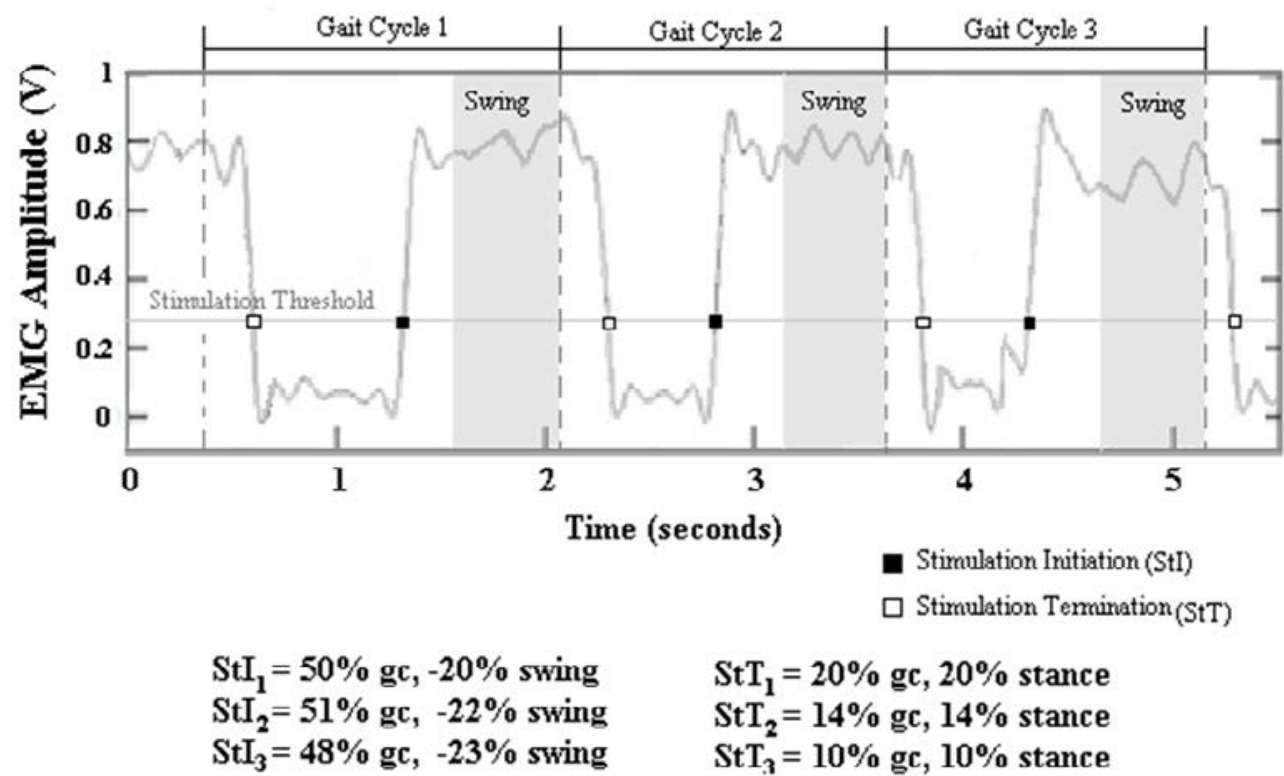

Figure 1: Sample stimulation initiation (Stl) and termination (StT) timing as defined in per cent gait cycle and relative to swing and stance, respectively.

\begin{tabular}{|c|c|c|c|c|c|c|c|}
\hline & Sex & Age (years) & Affected Side & Lower extremity Fugl-Meyer & Time since stroke (years) & Weight (kg) & Height $(\mathrm{cm})$ \\
\hline $\mathrm{S} 2^{*}$ & M & 51 & L & 27 & $4.5^{+}$ & 123.15 & 185.42 \\
\hline S4 & M & 48 & $\mathrm{R}$ & 23 & 7.5 & 75.07 & 185.42 \\
\hline S5 & M & 76 & L & 24 & 0.5 & 82.33 & 163.83 \\
\hline S6 & $\mathrm{F}$ & 65 & $\mathrm{R}$ & 23 & 2.5 & 60.21 & 154.94 \\
\hline $\mathrm{S} 7^{*}$ & M & 53 & L & 24 & 8 & 85.73 & 177.8 \\
\hline S8 & M & 51 & $\mathrm{R}$ & 21 & 2 & 126.32 & 180.34 \\
\hline S10 & $\mathrm{F}$ & 56 & L & 28 & 2 & 105.91 & 167.64 \\
\hline S12 & $M$ & 58 & L & 25 & $3.5^{++}$ & 119.29 & 177.8 \\
\hline Mean (sd) & & $57.3(9.2)$ & & (2.3) & $(2.7)$ & $97.3(24.8)$ & $174.1(10.9)$ \\
\hline
\end{tabular}

*study outliers, ${ }^{+}$initial stroke 9.5 years (mini stroke 4.5 years) prior to research participation,

${ }^{+}$initial stroke 1 month before stroke which caused foot drop, WA=WalkAide

Table 1: Subject Characteristics.

whether tilt or heel sensor-based stimulation is more robust during non-level walking. While improved stimulation reliability was observed for three subjects (S2, S6, S7) with the theoretical heel sensor-based stimulation, differences in stimulation reliability between sensors during non-level walking were not statistically significant.

Stimulation timing data for clinical tilt sensor-based control are summarized in Figure 3a. Timing outliers often existed due to extraneous stimulations, particularly for subjects S2 and S7. Omitting gait cycles with extraneous stimulations reduced much of the variability in the tilt sensor-based stimulation timing data as shown in Figure 3b.

For most subjects, stimulation initiation occurred at approximately $-20 \%$ swing, during the pre-swing phase of stance. Stimulation initiation was generally delayed as treadmill orientation progressed from declined to inclined, occurring later in stance. Post-hoc testing revealed statistically significant differences in stimulation initiation between declined/inclined treadmill orientations, with borderline statistical significance $(\mathrm{p}=0.023 \leq 0.05 / 3=0.017$ with Bonferroni correction) in stimulation initiation timing observed between level and declined orientations only.

For most subjects, stimulation termination occurred during the first $10-20 \%$ of the subsequent gait cycle, indicating that stimulation termination typically occurred during loading response of the subsequent cycle. Although differences in stimulation termination were observed between level and non-level walking for some subjects, no statistically significant differences in stimulation termination timing were observed between level and non-level ambulation.

Stimulation initiation and termination times were also estimated for theoretical heel sensor-based stimulation. These theoretical heel sensor-based stimulation initiation and termination data were again calculated both before and after eliminating outliers (Figure 4).

Stimulation initiation again occurred prior to swing. Although stimulation initiation occurred at approximately $-20 \%$ swings for tilt and heel sensor-based stimulations, theoretical heel sensor-based stimulation initiation demonstrated more consistent timing during non-level ambulation. No statistically significant differences in heel sensor-based stimulation initiation were observed between level and non-level walking trials. Additionally, no statistically significant differences in stimulation initiation timing between sensors were observed during non-level walking.

Stimulation termination for theoretical heel sensor-based stimulation also occurred during the subsequent stance period. These heel sensor-based stimulation termination times were slightly earlier than that observed during tilt sensor-based stimulation. No statistically significant differences in heel sensor-based stimulation termination 
Citation: Silver-Thorn MB, Gallagher MB, Long JT (2013) Effect of Tilt Sensor versus Heel Loading on Neuroprosthesis Stimulation Reliability and Timing for Individuals Post-Stroke during Level and Non-Level Treadmill Walking. Int J Phys Med Rehabil 1: 163. doi:10.4172/23299096.1000163

Page 4 of 7

(a)



(b) Heel Sensor (theoretically programmed)

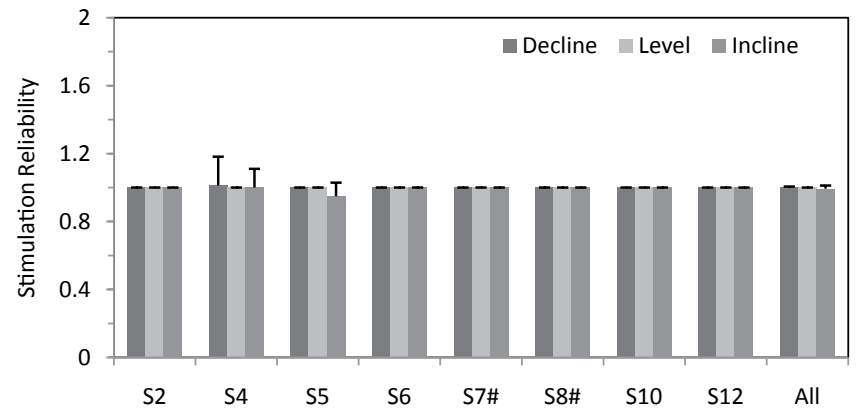

Figure 2: WalkAide stimulation reliability for tilt (a) and theoretical heel sensorbased (b) stimulation for all subjects for all treadmill orientations (approximately 30 gait cycles per subject per treadmill orientation). + denotes borderline statistically significant difference; \# reflects use of F-scan data rather than WalkAide heel sensor data for theoretical heel sensor- based stimulation.

were observed between level and non-level walking; differences in stimulation termination timing between sensors during inclined ambulation only demonstrated borderline statistical significance $(\mathrm{p}=0.051)$.

While extra stimulations occurred with both clinical tilt and theoretical heel sensor-based stimulations, fewer extra stimulations were observed for the theoretical heel sensor-based stimulation. As shown in Figure 5, the variability in stimulation initiation and termination timing was consistently less for theoretical heel sensorbased stimulation; these differences were statistically significant for stimulation termination during level and inclined walking only.

Post hoc power analysis for stimulation reliability and timing was also conducted. Although only 9 subjects are needed to detect significant differences in these parameters between sensors, more than 48 subjects are required to observe statistically significant differences in these parameters between level versus non-level walking.

\section{Discussion}

The WalkAide uses tibial tilt (or less commonly, heel loading) to define stimulation periods. Optimal stimulation reliability, one stimulation per gait cycle, is important to ensure the safety of individuals during neuroprosthesis use. Missed stimulations may result in little to no ankle dorsiflexion during swing, leading to insufficient toe clearance and increased fall risk. Extraneous stimulations may result in stimulations at random times throughout the gait cycle and no stimulation when most needed during swing.

Four of eight subjects demonstrated optimal stimulation reliability for all treadmill orientations using clinical tilt sensor-based stimulation.
Only one subject demonstrated stimulation reliability of 1.5 or greater, reflecting extraneous stimulations, for all treadmill orientations. This subject, however, walked significantly slower on the treadmill than overground. Since the WalkAide was programmed during faster overground walking, the programmed "wait time" (minimum time between successive stimulations) was likely insufficient for the slower treadmill ambulation, resulting in two stimulations during some gait cycles. The remaining subjects demonstrated both extraneous and missed stimulations for level and inclined treadmill orientations, and primarily missed stimulations for the declined treadmill orientation. Missed stimulations during declined walking were also reported by several subjects (S4, S6, S8) during the acclimation period.

Due to kinematic changes during non-level walking, stimulation reliability of tilt sensor-based stimulation was expected to be adversely affected by treadmill orientation. Borderline statistically significant differences in tilt sensor-based stimulation reliability were found between level and non-level walking. Extraneous and missed stimulations suggest that, depending on the individual's environment, clinicians should include both level and non-level walking during neuroprosthesis programming sessions. Three subjects (S8, S10, S12) who visited the clinician more frequently for neuroprosthesis adjustment exhibited consistent optimal stimulation reliability, suggesting that supplemental programming sessions may improve stimulation reliability during both level and non-level ambulation. The observed extraneous and missed stimulations are most likely due to changes in tilt sensor baseline values due to treadmill orientation [14]
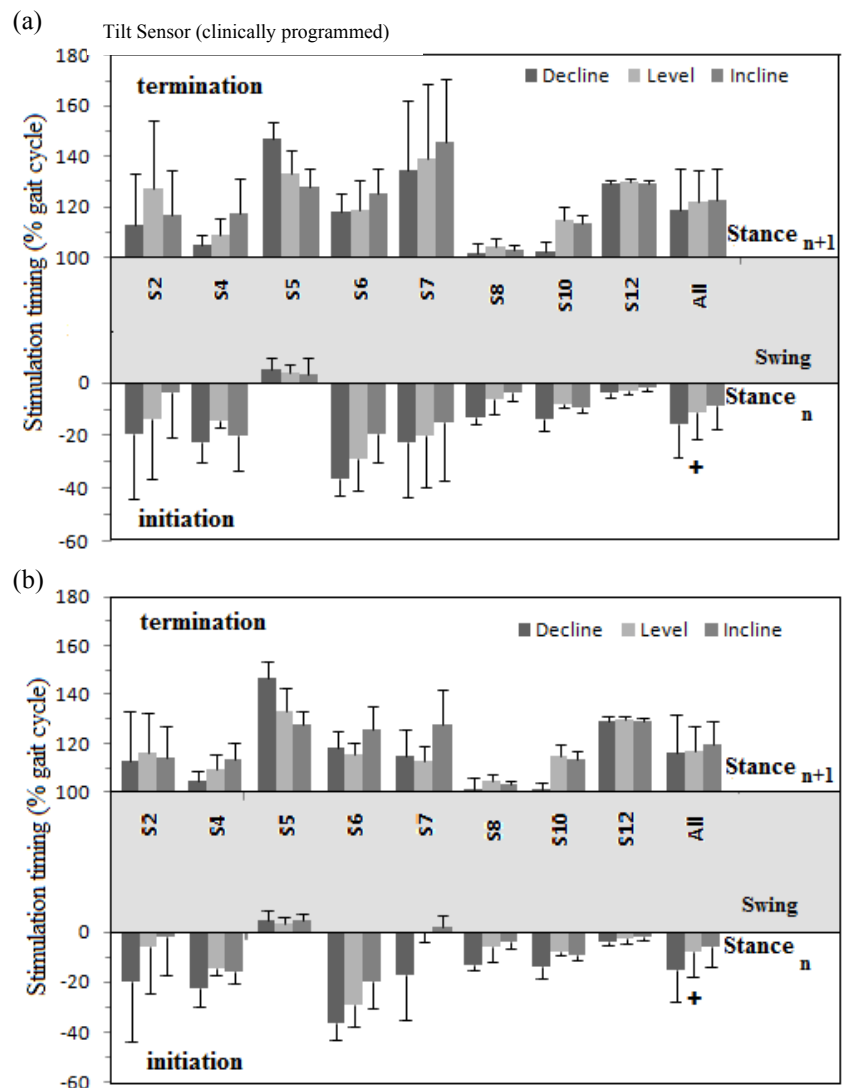

Figure 3: Tilt sensor-based stimulation timing before (a) and after (b) eliminating gait cycles with extraneous stimulations. Stimulation initiation occurs prior to swing, during the previous stance phase (negative \%cycle); stimulation termination occurs after swing, during the subsequent gait cycle (>100\% cycle). 
Citation: Silver-Thorn MB, Gallagher MB, Long JT (2013) Effect of Tilt Sensor versus Heel Loading on Neuroprosthesis Stimulation Reliability and Timing for Individuals Post-Stroke during Level and Non-Level Treadmill Walking. Int J Phys Med Rehabil 1: 163. doi:10.4172/23299096.1000163

(a) Heel Sensor (theoretically programmed)

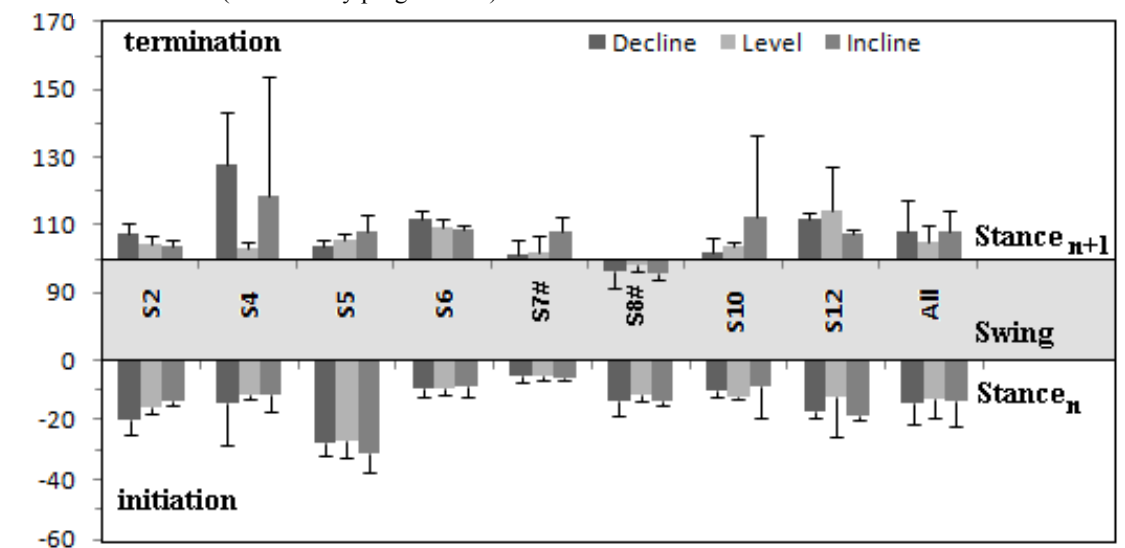

(b)

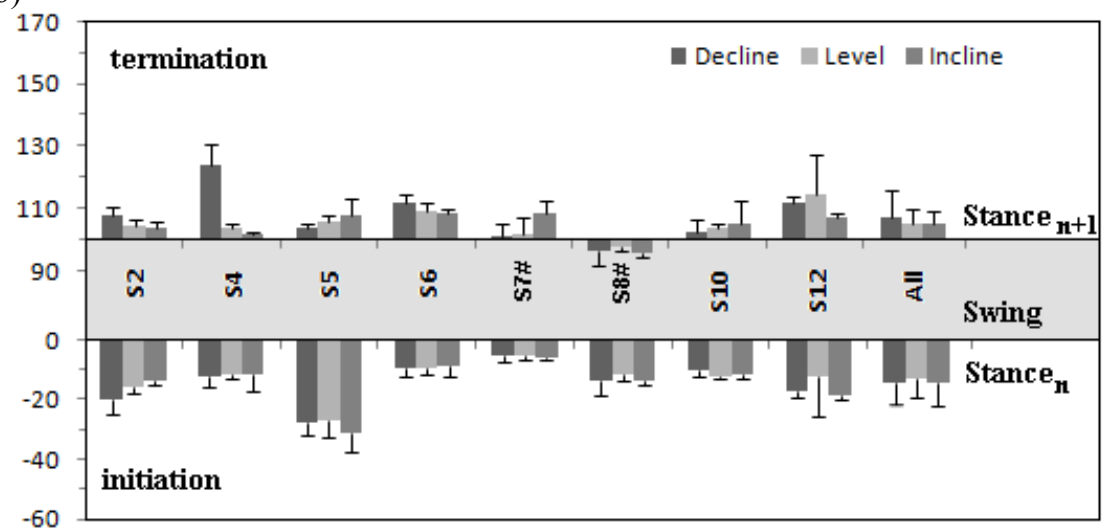

Figure 4: Theoretical heel sensor-based stimulation timing before (a) and after (b) eliminating gait cycles with extraneous stimulations. Stimulation initiation occurs prior to swing, during the previous stance phase (negative \%cycle); stimulation termination occurs after swing, during the subsequent gait cycle (> 100\% cycle). \# reflects use of F-scan data rather than WalkAide heel sensor data for theoretical heel sensor-based stimulation.

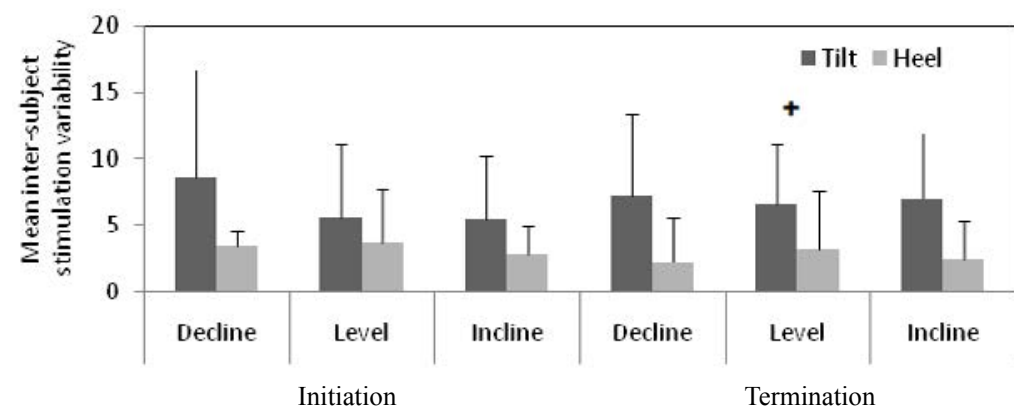

Figure 5: Clinical tilt and theoretical heel sensor-based stimulation timing variability for initiation (left) and termination (right)

as well as insufficient wait times between stimulations for the slower treadmill ambulation

Stimulation reliability of the theoretical heel sensor-based programming was unaffected by treadmill orientation; combined subject data resulted in stimulation reliability values of approximately one for all treadmill orientations. These results suggest that the heel sensor programming is not influenced by treadmill orientation, and may result in more robust stimulation reliability than for tilt sensorbased stimulation. Such conclusions, however, should be interpreted with caution as the theoretical heel sensor-based stimulation reliability results are dependent on the selected stimulation initiation and termination thresholds, as well as the assumed wait time and stimulation duration restrictions. In the current study, the initiation/termination thresholds were selected based on level treadmill ambulation; the wait time and stimulation duration were based on clinical programming during level overground walking (e.g. same values as for tilt-sensor based control).

No statistically significant differences in stimulation reliability errors were observed between sensors, suggesting that theoretical heel sensor-based stimulation was not more reliable than tilt sensor-based stimulation during non-level walking. Six of eight subjects exhibited optimal (unity) theoretical heel sensor-based stimulation reliability 
Citation: Silver-Thorn MB, Gallagher MB, Long JT (2013) Effect of Tilt Sensor versus Heel Loading on Neuroprosthesis Stimulation Reliability and Timing for Individuals Post-Stroke during Level and Non-Level Treadmill Walking. Int J Phys Med Rehabil 1: 163. doi:10.4172/23299096.1000163

Page 6 of 7

values and only four of eight subjects demonstrated optimal tilt sensorbased stimulation reliability values during non-level walking. As such, one might argue that the heel sensor may still be considered a better sensor for more reliable stimulation control during non-level walking, although further testing with more subjects and clinical heel sensorbased programming are needed before such preliminary findings can be substantiated.

Stimulation initiation was delayed as the treadmill orientation progressed from declined to inclined, occurring later in stance, closer to swing phase itself. The earlier stimulation initiation during declined walking may decrease plantar flexion during late stance, affecting forward progression of the affected limb during swing. Such plantar flexion activity may, however, be less necessary during declined walking as gravity assists with forward progression. Delayed dorsiflexion stimulation during inclined ambulation may result in decreased resistance to ankle plantar flexion during late stance facilitating push off and forward progression, and may therefore be advantageous.

Tilt sensor-based stimulation termination typically occurred during the first $10-20 \%$ of the subsequent gait cycle, indicating that anterior tibialis stimulation continued through loading response perhaps minimizing potential foot slap. Although differences in stimulation termination between treadmill orientations were observed for some subjects, no statistically significant differences in stimulation termination timing were observed between level and non-level walking.

The changes in stimulation initiation (but not termination) timing during non-level walking reflect prolonged stimulation duration during declined walking (and potential increased risk of anterior tibialis fatigue) and shorter stimulation periods during inclined ambulation.

Large variability in stimulation timing was observed, largely due to gait cycles with extraneous stimulations. This variability in stimulation timing may be attributed to tilt sensor errors (baseline changes with non-level walking, sensor movement and alignment errors) and/ or programming parameters (wait time, stimulation minimum or maximum duration). Stimulation timing variability was reduced for three subjects who had WalkAide programming adjustment one week prior to gait analysis.

For the theoretical heel sensor-based stimulation, stimulation initiation consistently occurred at approximately $-20 \%$ swing. Contrary to clinical tilt-sensor based stimulation, this initiation was not dependent on treadmill orientation and the potential benefit of delayed stimulation initiation during inclined ambulation was not present for heel sensor-based stimulation. Theoretical heel sensorbased stimulation termination occurred slightly earlier during the subsequent stance period than during tilt sensor-based stimulation, potentially reducing ankle stability during loading response to prevent foot slap.

The variability in stimulation timing was substantially less for heel sensor-based stimulation, most likely due to greater consistency in heel loading during level and non-level ambulation and perhaps the selection of stimulation initiation/termination thresholds based on level treadmill (not overground) ambulation. The heel sensor may therefore be considered more reliable for stimulation control during non-level walking. However, differences in tilt sensor stimulation initiation with treadmill orientation may actually improve the efficacy of tilt sensorcontrol, increasing the stimulation period during declined walking, providing controlled forward progression.

While prior investigations of neuroprostheses have been conducted for individuals post-stroke [6-12], these investigations involved level walking trails only and did not assess either stimulation reliability or stimulation timing. As such, the results of this study are novel and cannot be contrasted with the literature.

Both tilt and heel sensor-based stimulation reliability was likely adversely affected by the short wait time programmed during faster overground than treadmill ambulation. Another potential study limitation affecting stimulation reliability and timing was that the heel sensor-based stimulation reliability and timing data were based on theoretical programming using investigator-specified stimulation initiation/termination thresholds determined during level treadmill ambulation. For clinical tilt sensor-based programming, these thresholds were based on level overground ambulation. As the thresholds were based on level walking trials for both sensors, however, non-level walking may still affect sensor output contributing to differences in stimulation reliability and timing. Finally, the limited sample size (and perhaps variations in the number of programming sessions) likely affected the detection of potentially statistically significant differences between sensors and between treadmill orientations.

\section{Conclusions}

The stimulation reliability and timing of the WalkAide neuroprosthesis were contrasted for two programming options during level and non-level ambulation. Tilt sensor-based stimulation reliability differed significantly between level and non-level walking. The stimulation initiation timing during tilt sensor-based control occurred significantly closer to swing as the treadmill processed from declined to inclined orientations; this non-level stimulation initiation effect may be beneficial as stimulation initiation closer to swing during inclined ambulation may allow for greater ankle plantar flexion to assist with forward progression. Finally, although theoretical heel sensor-based stimulation control exhibited more consistent stimulation timing with less variability than for tilt sensor-based stimulation during nonlevel ambulation, no statistically significant differences in stimulation reliability or timing between sensors were observed.

Future study is recommended with an expanded subject population and clinical heel sensor-based programming to further investigate the effects of level versus non-level walking and stimulation control parameters on neuroprosthesis stimulation reliability and timing. These studies, and clinical practice, warrant more frequent programming refinement, particularly if the subject varies their cadence (as during treadmill ambulation) and/or encounters non-level terrain.

Such studies might also include kinetic data acquisition to investigate the effect of variations in stimulation initiation and duration on ankle moment and power during level and non-level ambulation.

\section{Acknowledgement}

The authors are grateful to Gerald Harris, Ph.D. for access to the gait laboratory and to Tina Stoeckmann, PT, Ph.D. and Andrew Starsky, PT, Ph.D. for their assistance with project definition, research hypotheses, and clinical relevance. Finally, the authors thank Daniel Eastwood, MS for his assistance with the statistical analyses. This project was supported by the Biomedical Engineering Department and the College of Engineering at Marquette University. The AFOs and neuroprostheses were provided by Hanger Prosthetics and Orthotics.

\section{References}

1. Lloyd-Jones D, Adams R, Carnethon M, De Simone G, Ferguson TB, et al. (2009) Heart disease and stroke statistics--2009 update: a report from the American Heart Association. Circulation 119: 480-486.

2. Wade DT, Wood VA, Heller A, Maggs J, Langton Hewer R (1987) Walking afte stroke. Measurement and recovery over the first 3 months. Scand J Rehabil Med 19: 25-30.

3. Hyndman D, Ashburn A, Stack E (2002) Fall events among people with stroke 
Citation: Silver-Thorn MB, Gallagher MB, Long JT (2013) Effect of Tilt Sensor versus Heel Loading on Neuroprosthesis Stimulation Reliability and Timing for Individuals Post-Stroke during Level and Non-Level Treadmill Walking. Int J Phys Med Rehabil 1: 163. doi:10.4172/23299096.1000163

Page 7 of 7

living in the community: circumstances of falls and characteristics of fallers. Arch Phys Med Rehabil 83: 165-170.

4. Sheffler LR, Chae J (2007) Neuromuscular electrical stimulation in neurorehabilitation. Muscle Nerve 35: 562-590.

5. The WalkAide Manual (2008) In: Neurotronics I, editor.

6. Laufer Y, Hausdorff JM, Ring H (2009) Effects of a foot drop neuroprosthesis on functional abilities, social participation, and gait velocity. Am J Phys Med Rehabil 88: 14-20.

7. Shimada $Y$, Matsunaga T, Misawa A, Ando S, Itoi E, et al. (2006) Clinical application of peroneal nerve stimulator system using percutaneous intramuscular electrodes for correction of foot drop in hemiplegic patients. Neuromodulation 9: 320-327.

8. Taylor PN, Burridge JH, Dunkerley AL, Wood DE, Norton JA, et al. (1999) Clinical use of the Odstock dropped foot stimulator: its effect on the speed and effort of walking. Arch Phys Med Rehabil 80: 1577-1583.

9. Burridge JH, Taylor PN, Hagan SA, Wood DE, Swain ID (1997) The effects of common peroneal stimulation on the effort and speed of walking: a randomized controlled trial with chronic hemiplegic patients. Clin Rehabil 11: 201-210.

10. Hausdorff JM, Ring H (2008) Effects of a new radio frequency-controlled neuroprosthesis on gait symmetry and rhythmicity in patients with chronic hemiparesis. Am J Phys Med Rehabil 87: 4-13.

11. Ring H, Treger I, Gruendlinger L, Hausdorff JM (2009) Neuroprosthesis fo footdrop compared with an ankle-foot orthosis: effects on postural control during walking. J Stroke Cerebrovasc Dis 18: 41-47.

12. Sheffler LR, Hennessey MT, Naples GG, Chae J (2006) Peroneal nerve stimulation versus an ankle foot orthosis for correction of footdrop in stroke: impact on functional ambulation. Neurorehabil Neural Repair 20: 355-360.

13. McIntosh AS, Beatty KT, Dwan LN, Vickers DR (2006) Gait dynamics on an inclined walkway. J Biomech 39: 2491-2502.

14. Gunther Gallagher M (2011) Comparison of an ankle-foot-orthosis and neuroprosthesis during level and non-level walking for individuals post-stroke. Master's Thesis, Marquette University.

15. Rice WR (1989) Analyzing tables of statistical data. Evolution 43: 223-225
Citation: Thorn BS, Gallagher MB, Long JT (2013) Effect of Tilt Sensor versus Heel Loading on Neuroprosthesis Stimulation Reliability and Timing for Individuals Post-Stroke during Level and Non-Level Treadmill Walking. Int J Phys Med Rehabil 1: 163. doi:10.4172/2329-9096.1000163
Submit your next manuscript and get advantages of OMICS Group submissions

Unique features:

User friendly/feasible website-translation of your paper to 50 world's leading languages

Audio Version of published paper

Digital articles to share and explore

Special features:

- 300 Open Access Journal

25,000 editorial team

21 days rapid review process

- Quality and quick editorial, review and publication processing

- Indexing at PubMed (partial), Scopus, EBSCO, Index Copernicus and Google Scholar etc

- Sharing Option: Social Networking Enabled

- Authors, Reviewers and Editors rewarded with online Scientific Credits

- Better discount for your subsequent articles

Submit your manuscript at: http://www.omicsonline.org/submission/ 